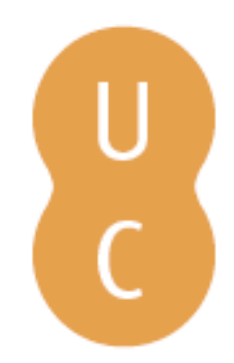

\title{
pompalina
}

\section{Espacios para la curación: la domus en la tradición hipocrático-galénica}

Autor(es): $\quad$ Fernández Tijero, Mํㅡㄹ Carmen

Publicado por: Associação Portuguesa de Estudos Clássicos; Imprensa da

Universidade de Coimbra

URL

persistente: URI:http://hdl.handle.net/10316.2/31757

DOI: $\quad$ DOI:http://dx.doi.org/10.14195/978-989-721-069-3_11

Accessed : $\quad$ 26-Apr-2023 15:19:19

A navegação consulta e descarregamento dos títulos inseridos nas Bibliotecas Digitais UC Digitalis, UC Pombalina e UC Impactum, pressupõem a aceitação plena e sem reservas dos Termos e Condições de Uso destas Bibliotecas Digitais, disponíveis em https://digitalis.uc.pt/pt-pt/termos.

Conforme exposto nos referidos Termos e Condições de Uso, o descarregamento de títulos de acesso restrito requer uma licença válida de autorização devendo o utilizador aceder ao(s) documento(s) a partir de um endereço de IP da instituição detentora da supramencionada licença.

Ao utilizador é apenas permitido o descarregamento para uso pessoal, pelo que o emprego do(s) título(s) descarregado(s) para outro fim, designadamente comercial, carece de autorização do respetivo autor ou editor da obra.

Na medida em que todas as obras da UC Digitalis se encontram protegidas pelo Código do Direito de Autor e Direitos Conexos e demais legislação aplicável, toda a cópia, parcial ou total, deste documento, nos casos em que é legalmente admitida, deverá conter ou fazer-se acompanhar por este aviso.

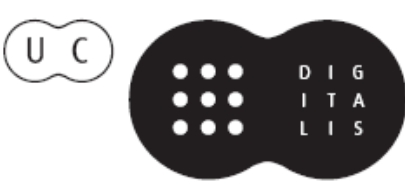




\section{Espaços e Paisagens}

\section{Antiguidade Clássica e Heranças Contemporâneas}

Vol. I Línguas e Literaturas. Grécia e Roma

Francisco de Oliveira, Cláudia Teixeira, Paula Barata Dias (coords.)

IMPRENSA DA UNIVERSIDADE DE COIMBRA 


\title{
ESPACIOS PARA LA CURACIÓN: LA DOMVS EN LA TRADICIÓN HIPOCRÁTICO-GALÉNICA
}

\author{
Ma Carmen Fernández Tijero \\ Universidad de Valladolid
}

\begin{abstract}
From the very beginning of the development of curative methods in ancient Roman society we can observe a special union between a determined space and healing abilities, as is known about the history of a kind of clinical assistence on the Tiberine Island. There were different places devoted to the healing of illnesses in the ancient Roman civilization: the tabernae medicae, both private and public assistence, Roman soldiers set up their valetudinaria in the base-camps during their conquests; as well as they were called valetudinaria the medical assistence rooms in the country properties. However, we shall pay special attention to the space of the domus as a place where the doctor examines, diagnoses, treats and heals the sick, and furthermore we can see how these practitioners attend as something relevant for the healing procedure not only the symptoms of the sick body, but also the space where this sick body is: the materials, orientation, distribution, etc. Roman builders and architects did follow strict rules of hygiene in examining the place where they were building; Vitrubio`s work De architectura provides the best example of these precepts. At this point, the medieval medical texts which follow the HippocraticGalenic tradition, and develop it at the incipient European universities, give us the evidence for the importance of the space for healing in the ancient Roman and the european medieval civilizations. We may observe appearance of a practical genre of texts treating the medical visit in the domus step by step. Among them, we examine the Modus medendi and De ingenio sanitatis by Bernard de Gordon, professor and practitioner at the University of Montpellier in the XIV century.
\end{abstract}

Keywords: Avicena, Bernardo de Gordon, De ingenio sanitatis, De modo medendi, domus, Galeno, hospital, insula, Montpellier, Roman architecture, valetudinaria, villa.

Palavras-chave: arquitectura romana, Avicenas, Bernardo de Gordon, De ingenio sanitatis, De modo medendi, Galeno, hospital, insula, Montpellier, valetudinaria, villa.

El objetivo de este trabajo es presentar una idea clara de los espacios dedicados a la curación en la civilización romana y en las culturas europeas, continuadoras de ésta, atendiendo especialmente a la domus. Acudimos a dos tipos de fuentes: los restos arqueológicos y los textos, tanto literarios en general, como tratados más específicos de tipo científico-técnico, que son los 
que ponen de relieve la importancia del espacio doméstico en el proceso de diagnóstico y tratamiento médico.

\section{Lugares para la práctica de la medicina}

La unión de los procesos curativos con un lugar concreto está ya presente en la propia llegada de la medicina a Roma, con la leyenda que cuenta cómo arraigó el culto a Esculapio con un templo en la Isla Tiberina y establecimientos en torno a dicho templo donde los enfermos practicaban la incubatio para recobrar la salud ${ }^{1}$.

Entre los primeros lugares concretos dedicados a la medicina de los que tenemos mención en las fuentes literarias y restos arqueológicos están las tabernae, término latino que designa el tipo de establecimiento artesanal, en locales abiertos a la calle en las fachadas de los edificios². Después llamados taberna medica, y posteriormente medicina. En las representaciones de los estudios de los médicos podemos apreciar su distribución: con estanterías con libros, una mesa y algunos utensilios médicos, pero bastante sencillas. También se vendían preparaciones médicas y cosméticos, y eran además una especie de puestos de socorro y casas de $\operatorname{cura}^{3}$, una especie de clínicas privadas donde se hospitalizaban y curaban a las órdenes de un médico que tenía a su disposición asistentes o mediastini, y distintos enfermeros, como masajistas o reunctores ${ }^{4}$. Podían ser consultas privadas o de propiedad pública, donde trabajaba el archiater municipal ${ }^{5}$.

En cuanto a los médicos estatales, sabemos con certeza que el estado aseguraba la asistencia médica en diversos lugares concretos de las ciudades, existía un médico dedicado a los jardines públicos, otro, evidentemente necesario, en el anfiteatro, en las bibliotecas, un archiater portus en Ostia, etc ${ }^{6}$. Incluso algunos hablan ya de la existencia de leproserías ${ }^{7}$. Existían también ambulatorios, aunque su arquitectura no se diferencia de la de otros edificios, y no estaban dotados de instalaciones fijas, por lo que sólo los materiales médicos pueden ayudar a distinguirlos. Los útiles hechos con madera: tablas, registros, etc., no se han conservado por la fragilidad del material en sí mismo, y los instrumentos quirúrgicos, de materiales más duraderos, no se solían abandonar en los edificios por su gran valor; por lo que carecemos de cantidad de información. En Pompeya, gracias al buen estado de

${ }^{1}$ Narran la historia Tito Livio, 10,47,7; Valerio Máximo, 1,8,2; Estrabón, Geographica, 12,567; Plutarco, Quaestiones Romanae, 94; y Ovidio, Metamorphoseon libri, 15, 622-745. Cf. M. Besnier (1902), M. Guarducci (1971).

${ }^{2}$ Cf. Plauto, Amphytruo, 1011-1013. Fedro en su fábula 148 habla de las tabernae y los iatreia griegos.

${ }^{3}$ Cf. Cicerón, Pro Cluentio, 178; Plauto, Mercator, 471-472.

${ }^{4}$ Cf. Plinio, op.cit., $29,2$.

${ }^{5} \mathrm{El}$ primer iatreion público en Roma fue comprado por los senadores, a espensas del estado, para el médico griego Arcágato. Cf. Plinio, op.cit., 29,6.

${ }^{6}$ Cf. J. André 1987: 109.

${ }^{7}$ Cf. J. André 1987: 117. 
conservación de los edificios y sus haberes, podemos situar la "Casa del Cirujano", por los utensilios encontrados en el suelo del salón.

Otro tipo de establecimiento de asistencia médica son los valetudinaria. Bajo esta denominación encontramos una especie de enfermerías privadas, las clínicas de las grandes haciendas agrícolas, donde se trataban los esclavos; y, un tipo concreto de valetudinaria mejor conocidos son los militares 9 . Éstos eran parte integrante del campamento romano, construidos un poco apartados para asegurar la tranquilidad, y la planta arquitectónica de su edificio era inconfundible: tenía varios ambientes dispuestos en torno a una estancia central, un patio, y entre ellos había habitaciones para el reposo de los enfermos, habitaciones de aislamiento, salas de operaciones, cocina, baños, etc. El ejemplo más completo lo tenemos en el hospital legionario de Castra Vetera (hoy Xanten), en Alemania, de tiempos de Nerón.

En Roma se echaban en falta hospitales civiles, hecho que muestra su mentalidad pragmática: los hospitales militares y de las haciendas agrícolas para el cuidado de los esclavos satisfacían ya su carácter utilitario. Habrá que esperar al triunfo del Cristianismo para que se extiendan por todo el territorio del Imperio hospitales civiles, a partir de los llegados de Oriente. Surgen entonces diferentes tipos de espacios para la asistencia médica, hay nosocomia (hospitales); orphanotrophia (orfanatos), ptochotrophia (asilos para los pobres), gerontocomia (asilos para los ancianos), y brephotrophia (hospicios para los niños), mantenidos todos ellos por el erario público ${ }^{10}$.

Pero si hay algo que defina la asistencia médica en la civilización romana es la visita del médico en la propia casa del enfermo.

\section{El espacio de la domus}

Todo espacio en la civilización romana tenía una distribución funcional. La casa de los romanos refleja en todo momento su vida en sociedad y su forma de vida privada. Hay tres tipos de viviendas:

- domus, vivienda unifamiliar de tipo señorial;

- insula, edificios de varias plantas en las grandes ciudades donde vivían la mayoría de los ciudadanos en alquiler, en condiciones de comodidad e higiene nada deseables ${ }^{11}$.

- viviendas en el campo: se diferencian las viviendas de las familias humildes de labradores, casae o tuguria, las fincas de los ricos terratenientes, fundi, y sus casas de recreo o villa.

\footnotetext{
${ }^{8}$ Aparecen por primera vez en la literatura latina en Catón, De agricultura 2,2, y son descritos por Columela en el capítulo 11 de su tratado De agricultura o De re rustica.

${ }^{9}$ Tenemos, entre otras, las descripciones de Higinio, De munitionibus castrorum, 4.

${ }^{10}$ Cf. Codex Justinianus, 1,3,42,13-20.

${ }^{11}$ Los emperadores romanos emitieron diversos decretos para delimitar la altura de los edificios, porque se llegó a alturas excesivas. Trajano fijó la altura máxima en 60 pies, i.e., 17,8 metros. Cf. A. Dosi-F. Schnell 1992: 49.
} 
De estos tres tipos de vivienda, la asistencia médica $\epsilon \mathcal{L} \nu \lambda \iota \nu \eta ́$, la que se realizaba en la cabecera de la cama del enfermo, era la propia de un nivel social medio-alto, por lo que tomamos como lugar ejemplar para la visita médica la domus. Los miembros de las clases altas romanas, en cualquier parte del imperio, podían hacerse construir una casa siguiendo una tipología establecida. Más que su distribución interior, nos interesa ver su relación con la medicina. En primer lugar, sabemos que la domus es el final del desarrollo espacial urbano de la cabaña primitiva, que también dio lugar al nacimiento de las villas, en el terreno rural. En la delimitación del espacio doméstico de la cabaña, éste se liberaba de posibles fuerzas oscuras o malas influencias. Otro aspecto esencial era la higiene de los lugares donde se iba a realizar cualquier tipo de construcción ${ }^{12}$, son varios los autores clásicos que dan recomendaciones higiénicas para la construcción de las ciudades en general y del espacio doméstico en particular ${ }^{13}$. El mejor testimonio lo ofrece el texto de Vitrubio, De architectura, organizado en diez libros, de los cuales el sexto se dedica a los espacios privados, y del que podemos extraer las siguientes consideraciones: Hay que tener en cuenta la latitud y la orientación de los edificios que van a construirse, según las peculiaridades de la región y las condiciones del clima ${ }^{14}$. Tras determinar las medidas y proporciones de las casas y sus compartimentos, dedica los capítulos cuarto y quinto a aspectos pertinentes de las distintas salas y la disposición más conveniente de las casas, según la escala social. En relación con la visita médica, de estos capítulos nos interesa destacar que los dormitorios y las bibliotecas deberán orientarse hacia el este, ya que el uso de estas estancias exige la luz del amanecer ${ }^{15}$; y conviene distinguir las habitaciones destinadas al público de las privadas. De modo que los personajes importantes de la sociedad deben cuidar de mantener su estatus social en las salas donde reciben a gente, y evitar recargar las privadas.

\section{La visita médica}

Cuando los médicos eran llamados para asistir a los enfermos en casa ${ }^{16}$, ÉV K $\lambda \chi^{\prime} \nu \eta n$, "a la cabecera de la cama”, toma el nombre de clinicus $^{17}$. Después se le llamará visitator, y, por extensión, la visitatio llega a ser sinónimo de tratamiento. Esta medicina clínica se consideraba una rama particular de la

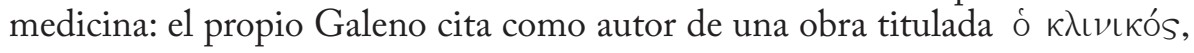

${ }^{12}$ Aspecto que ya determinó la ubicación del templo a Esculapio en la Isla Tiberina, dejando de lado la leyenda.

${ }^{13}$ Columela, op.cit.,1,5; Varrón, De agricultura 1,12; Catón, De agricultura, 3; 4; 10.

${ }^{14}$ Vitrubio, op.cit., 6,1.

${ }^{15}$ Vitrubio, op.cit., 6,4.

${ }^{16}$ Varrón nos da una lista de verbos que se usaban para llamar al médico: accersere, adducere, adhibere, convocare, advocare, vocare, quaerere, rogare (op.cit., 1,69,3).

${ }^{17}$ CIL 6,2532; J. André 1987: 76. 
El médico de los que están en cama, a un tal Damócrates ${ }^{18}$. Así se comienzan a escribir obras dedicadas específicamente a esta visita médica en casa.

Entre los textos médicos que tratan este tema destacan en la antigüedad grecolatina los maestros Hipócrates y Galeno ${ }^{19}$ y, de una manera más concreta, aparecen en la Edad Media un conjunto de obras que podemos englobar en un subgénero literario que analizan todos sus elementos, desde el comportamiento ético del médico hasta el de sus asistentes, el enfermo, y uno a uno de los pasos a seguir por el clínico en esta primera visita. Son tratados breves de tipo práctico que deben entenderse en el marco de la incipiente enseñanza universitaria y que recogen la doctrina de las auctoritates clásicas Hipócrates y Galeno, y de los brillantes científicos árabes, como Avicena. Presentamos como ejemplo de esta tradición dos obras del maestro de la Universidad de Montpellier Bernardo de Gordon -segunda mitad del siglo XIII- que combina la metodología escolástica de respeto y veneración por las auctoritates medicae que suponían Hipócrates y Galeno con un marcado carácter práctico.

Son: el tratado titulado De ingenio sanitati ${ }^{20}$, De decem ingeniis curandorum morborum, o Methodus medendi, basado en la obra de Galeno que lleva el mismo título y en Avicena, y el más breve De modo medendi.

En el primer tratado el autor medieval expone el método de cura de las enfermedades, desde cómo debe estar orientada y ordenada la casa del enfermo hasta el tratamiento adecuado según el diagnóstico que el físico haya realizado, para el que también da indicaciones. Sigue una organización muy clara, dividiendo su obra en diez métodos; en el primero analiza varios aspectos, entre ellos los cambios y movimientos, procurando buscar siempre un entorno favorable física y psicológicamente al enfermo. En este entorno debe atender a la casa, la ciudad, la habitación y sus alrededores, y los asistentes ${ }^{21}$. Debe cambiar de una casa a otra, de una habitación a otra, de grandes a pequeñas ciudades, a zonas verdes, montes, valles, aguas, fuentes y aire limpio, y cuidar de que todo procure el deleite y un ambiente más puro. Principios ya expuestos por Avicena. ${ }^{22} \mathrm{Un}$ poco más adelante, en el octavo método, insiste en la importancia del ambiente saludable, y la importancia del espacio donde el enfermo se encuentra para lograr su curación, siendo determinantes incluso los materiales de construcción de la domus, para adecuarse lo más posible al clima en que se encuentre, como hemos

${ }^{18}$ Cf. Galeno, Opera Omnia, 13,349; Plinio, op.cit., 29.4.

${ }^{19} \mathrm{De}$ Galeno destaca en este tema los tratados Methodus medendi, Liber prognosticorum y Ars medica, entre otros; Hipócrates expone sus ideas acerca de el tema que nos ocupa en sus obras Aphorismi, Regimen acutarum egritudinum y Epidemiarum libri, entre otras.

${ }^{20}$ La expresión ingenium sanitatis o ingenium curationis designa, a partir de finales del siglo XIII, el paso que se da en la terapéutica médica de los principios teóricos a la puesta en práctica de un tratamiento específico, teniendo en cuenta todos los factores relativos a la enfermedad, a la constitución patológica del enfermo, a su modo de vida, su lugar de residencia, etc, en fin, todo lo que pueda influir en el tratamiento y la cura de la enfermedad en ese caso concreto.

${ }^{21} \mathrm{Cf} . \mathrm{M}^{\mathrm{a}} \mathrm{C}$. Fernández Tijero, De decem ingeniis curandi morbos y la literatura de Modus medendi atribuida a Bernardo de Gordon. Estudio, edición crítica y traducción, Tesis Doctoral, 2007, Universidad de Valladolid, en prensa, 2, 3, 1.36-39.

${ }^{22}$ Cf. Canon 1 ,fen 4,68vb. 
visto que ya anunciaba Vitrubio. Es importante también la ubicación de la casa en un entorno tranquilo, sin tener cerca talleres ruidosos, o donde se trabaje con minerales, y que la habitación del enfermo esté refugiada, no expuesta a fuertes vientos ${ }^{23}$.

El segundo de ellos, el breve De modo medendi, con una finalidad didácticopráctica, ofrece una serie de consejos para que el médico, aún inexperto o con poca práctica, sepa qué hacer en esa primera visita al enfermo, cómo diagnosticar la enfermedad, qué elementos debe analizar, entre los que está la disposición del ambiente que rodea al enfermo, cómo dirigirse a éste y a sus familiares para que confíen en él, y qué tratamiento debe prescribir según los síntomas presentados. Establece también un orden estricto en la terapéutica: primero un régimen en su dieta y modo de vida en general, donde se ubicarían los cambios de ambiente que refiere en la obra citada anteriormente; después la prescripción de medicinas y, por último, y sólo cuando ninguno de estos dos tipos de tratamiento no haya funcionado, la intervención quirúrgica ${ }^{24}$.

\section{Conclusión}

En estos textos queda clara la relevancia del espacio donde se ubica el enfermo, que se puede resumir en dos aspectos:

- por una parte, en el momento de construcción de los espacios domésticos, se buscaba un lugar idóneo para la salubridad y se expulsaba toda posible influencia negativa sobre ese lugar concreto mediante ceremonias religiosas de tipo apotropaico ${ }^{25}$;

- por otra parte, es esencial en el examen médico, ya que la orientación misma de la casa, su disposición, aireación, etc. influyen en la evolución de la enfermedad y, por consiguiente, en el desarrollo del tratamiento.

Dos aspectos que muestran que la unión entre espacio y capacidad de curación sigue estando presentes, como veíamos ya en el momento de la llegada de la medicina a Roma, en la Isla Tiberina.

\section{Bibliografía}

\section{Textos}

Avicena, Liber canonis. Hildesheim-Zürich-New York. 2003. Catón, De agricultura. Paris, Les Belles Lettres. 1975.

${ }^{23}$ Cf. $M^{a}$ C. Fernández Tijero, op. cit., 2,3,1. 55-65.

${ }^{24}$ Cf. Ma C. Fernández Tijero, op. cit., 3,2,1. 3-6; 17-22; 64-72.

${ }^{25}$ Recordemos, por ejemplo, que al nacer un niño, su padre y otros hombres de la casa se colocaban a la puerta para expulsar a Silvanus. Hecho que reflejaba el miedo a que un espíritu maligno se lleve la vida del niño y de la madre. Una vez más el espacio doméstico es un lugar protegido, delimitado. 
Cicerón, Orationes. Paris, Les Belles Lettres. 1929-1989.

Columela, De agricultura. Paris, Les Belles Lettres. 1969-1993.

Corpus Inscriptionum Latinarum. Berolini, Weidmannos, 1841-1849.

Estrabón, Geographica. Paris, Les Belles Lettres 1966 sq.

Fedro, Fabulae. Paris, Les Belles Lettres. 1969.

Galeno, Opera Omnia, Kühn (ed.) I-XXII. Leipzig 1821-1833 (reimpr. 19641965).

Higinio, De munitionibus castrorum. Paris, Les Belles Lettres. 1979.

Codex Iustinianus, Berolini, Weidmannos, 1900.

Ovidio, Metamorphoseon libri. Barcelona, Alma Mater. 1964-1994.

Plauto, Comoediae. Oxonii, Typographeo Clarendoniano. 1991.

Plinio, Naturalis Hstoria. Paris, Les Belles Lettres, 1950-1981.

Plutarco, Quaestiones Romanae. Paris, Les Belles Lettres. 2002.

Tito Livio, Ab urbe condita. Paris, Les Belles Lettres. 1946 sq.

Varrón, De agricultura. London, The Loeb Classical Library, 1960.

Valerio Máximo, Factorum et dictorum memorabilium libri. Paris, Les Belles Lettres. 1995-1997.

Vitrubio, De architectura. Darmstadt, Wissensc Buchg. 1987.

\section{Bibliografía General}

J. André (1987), Etre médecin à Rome. París.

M. Besnier (1902), "L'ile Tiberine dans l'antiquitè", en BEFAR. París.

A. Dosi, F. Schnell (1992), "Spazio e Tempo", Vita e costumi dei romani antichi 14. Roma.

Ma C. Fernández Tijero, "De decem ingeniis curandi morbos" y la literatura de "Modus medendi" atribuida a Bernardo de Gordon. Estudio, edición critica y traducción, Tesis Doctoral, 2007. Universidad de Valladolid, en prensa.

M. Guarducci (1971), "L'Isola Tiberina e le sue tradizioni ospitaliere”, $R A L$ 27 267-281. 